\title{
Knowledge repository framework for crowdsourcing innovation intermediary: a proposal
}

\author{
Cândida Silva ${ }^{1}$ and Isabel Ramos ${ }^{2}$ \\ 1 School of Management and Industrial Studies, Polytechnic Institute of Oporto, Rua D. \\ Sancho I, 981, 4480-876 Vila do Conde, Portugal \\ candidasilva@eu.ipp.pt \\ 2 Department of Information Systems, University of Minho, Campus de Azurém, 4800-058 \\ Guimarães, Portugal \\ iramos@dsi.uminho.pt
}

\begin{abstract}
A crowdsourcing innovation intermediary performs mediation activities between companies that have a problem to solve or that seek a business opportunity, and a group of people motivated to present ideas based on their knowledge, experience and wisdom, taking advantage of technology sharing and collaboration emerging from Web2.0. As far as we know, most of the present intermediaries don't have, yet, an integrated vision that combines the creation of value through community development, brokering and technology transfer. In this paper we present a proposal of a knowledge repository framework for crowdsourcing innovation that enables effective support and integration of the activities developed in the process of value creation (community building, brokering and technology transfer), modeled using ontology engineering methods.
\end{abstract}

Keywords: open innovation, crowdsourcing innovation, knowledge repository, ontologies.

\section{Introduction}

Globalization, the developments of the internet and of the technology in general, are being the leverage to new ways of people to communicate and interact. This new world and new markets, in a daily change, are forcing enterprises to pay more attention to costumers needs, and therefore, to become more competitive and innovative. To face all these challenges, companies can not only depend on their internal skills [1].

Chesbrough argues that a company many times invests in $R \& D$ that turns out to be of no use to it, but that doesn't means that founding would be useful to other company of the same industry or even from another industry sector. Additional profit can be made from commercializing internal and external R\&D, taking advantage of the abundant knowledge that already exists in the market [2].

Open innovation is a new paradigm that proposes the use of external and internal ideas, and internal and external paths to market, as means to reach advances in technology used by companies [3]. 
Open innovation is thought to bring a number of benefits such as faster time to market for products, access to unique knowledge external to the organization, less cost of innovation, better adaptation of products and services to customer needs, commercial utilization of knowledge or technology that otherwise would have been wasted, shared risk in product and service development, and enhanced company image and reputation. The ideas and expertise can be found outside a company's boundaries and exported from within, and can create significant value for the company [4].

Collaborative software is being used by all kind of people and for all kind of tasks, from entertainment to business. Information, knowledge, experience, wisdom is already available in the millions of the human beings of this planet, the challenge is to use them through a network to produce new ideas and tips that can be useful to a company with less costs. The knowledge within a crowd and its capability to best solve problems than an individual, even an expert, is a subject that has been studied since the beginning of the nineteen century. Since then, there are many examples and demonstrations that, the probability of a heterogeneous crowd to best solve a problem is higher than an expert of the area [1]. The World Wide Web facilitates this kind of contributions, opening space to the emergence of crowdsourcing innovation initiatives.

Crowdsourcing innovation is a way of using the Web 2.0 tools to generate new ideas through the heterogeneous knowledge available in the global network of individuals highly qualified and with easy access to information and technology [5]. Adams and Ramos [6] discuss crowdsourcing innovation as a promising way of innovation outsourcing, especially for MSME's.

Ramos et al. [7] presented a conceptual crowdsourcing innovation intermediary model that integrates three modules in the process of creating value to a company: community development, brokering and technology transfer.

In this research work, we propose a conceptual knowledge repository framework for crowdsourcing innovation intermediaries composed by those three modules. This framework is being modeled using ontology engineering methods.

Ontologies are presented as a conceptual model for the systematization and formalization of knowledge in a particular area of knowledge. This conceptualization is rendered concrete with the definition of terms and concepts from the domain of knowledge in analysis, their relationships, organization and hierarchy, and allows the sharing and reuse by different people and systems of such knowledge [8-11].

This paper is organized in 5 sections. In the introduction was justified this research work presenting open innovation and crowdsourcing innovation concepts. The next section characterizes crowdsourcing innovation intermediaries and their business models. After, it is made a literature review of the ontology subject, its types and application areas. In section four we present a proposal of a conceptual knowledge repository for crowdsourcing innovation intermediaries which integrates the knowledge created by the community, the activities develop in the innovation brokering and in the technology transfer. Finally we made some conclusions of the research done and present the future work. 


\section{Crowdsourcing innovation intermediaries}

In June of 2006, Jeff Howe, a US journalist, introduced the term crowdsourcing, in an article in Wired Magazine [12], as a way of using the Web 2.0 tools to generate new ideas through the heterogeneous knowledge available in the global network of individuals highly qualified and with easy access to information and technology. Although, this concept has been used a quite time, the creation of the Wikipedia and of many examples of free software, like Linux, are examples of crowdsourcing activity.

Howe [5] breaks crowdsourcing into four models: collective intelligence or crowd wisdom, crowd creation, crowd voting and crowd funding, laying out examples that businesses can tailor to their own circumstances. Although, noting that successful crowdsourcing project often use a combination of these approaches.

Innovation intermediaries are organizations or groups within organizations that work to enable innovation, either directly by enabling the innovativeness of one or more firms, or indirectly by enhancing the innovative capacity of regions, nations, or sectors. Intermediaries are more than simple agents or brokers that just act as broker or agent between two or more parties. They are also engaged in other activities. Intermediaries' activities can be classified into three categories: interorganizational networking activities, technology development and related activities, and other activities [12].

A crowdsourcing innovation intermediary is an organization that mediates the communication and relationship between the seekers - companies that aspire to solve some problem or to take advantage of any business opportunity - with a crowd that is prone to give ideas based on their knowledge, experience and wisdom.

For crowdsourcing innovation intermediary the crowd is composed by groups of specialists in different areas, such as individual researchers, research team, labs, postgraduate students and highly qualified individuals.

It has being appearing some crowdsourcing innovation brokers, for example, Innocentive, yet2.com, Nine Sigma, IdeaConnection, some focus their business model in community development, others in brokering and others on technology transfer.

Ramos et al. [7] proposed a model that integrates these three modules in the process of creating value, and call them (1) knowledge network, (2) innovation brokering, and (3) innovation incubator. The module (1) integrates activities related with knowledge transfer to the network by each participant, the knowledge construction through the network, and the community building. The second module takes care of the knowledge management related with tasks like companies' needs and challenges proposals, the intellectual property management, and the innovation incubator has activities of consultancy, technology observatory, and funding opportunity tracking. They justify that for MSMEs the need for an integrated service is even more pressing. 


\section{Ontologies}

Ontologies have proliferated in the last years, mostly in Computer Science and Information Systems areas. This is essentially justified by the need of achieving a consensus in the multiple representations of reality inside computers, and therefore the accomplishment of interoperability between machines and systems [8].

There are several definitions of the concept of ontology from where can be assemble that it has an informal and formal notion associated to it. Gruber [9] definition clearly shows these - "An ontology is a formal, explicit specification of a standard conceptualization".

\subsection{Definition}

An ontology is a conceptualization of world view with respect to a given domain. This world view is conceived by a framework as a set of concept definitions and their interrelationships, that may be implicit, existing only in someone's head or tool, or explicit which includes a vocabulary of terms and a specification of their meanings.

The specification of that world view by means of a formal and declarative representation, with semantic interconnections, and some rules of inference and logic, will perform the formal ontology. The formal representation will facilitate the interoperability between heterogeneous machines and systems.

Ontologies have been developed with the promise of providing knowledge sharing and reuse between people and systems, by building a conceptual framework of a given knowledge domain to be represented. This framework will be formalized through a specific ontology language which will clearly express a controlled vocabulary and taxonomy, enabling the knowledge sharing and reuse.

The vocabulary is a list of terms or classes of objects, respective definitions and relationships between each other, provided by logical statements. They also specify rules for combining the terms and their relations to define extensions to the vocabulary.

The taxonomy or concept hierarchy is a hierarchical classification or categorization of entities in the domain of an ontology. The taxonomy should be in a machinereadable and machine-processable form in order to permit interoperability.

The full specification of an ontology domain establishes a conceptual framework, composed by the vocabulary and the taxonomy, for discussion, analysis, and information retrieval in a domain.

Ontology development requires an effective ontological analysis of the content the world view domain that it intends to represent. This analysis will reveal the terms and concepts of the domain knowledge, their relations, organization and hierarchy. Thus, they clarify the structure of domain knowledge, so, it can be called a content theory [10].

As the objective of ontologies is to facilitate knowledge sharing and reuse between various agents, regardless of whether they are human or machines, then it can be said that ontologies are a prerequisite and a result of a consensual point of view on the world. It is a prerequisite for consensus because to have knowledge sharing agents must agree on their interpretation of a domain of the world. And it is a result of 
consensus because the model of meanings was built as result of a process of agreement between agents on a certain model of the world and its interpretations. Therefore, it is an essential requirement that any ontology can progress over the time [11].

Briefly, an ontology provides an explicit conceptualization that describes the semantics of the data. As Fensel [11] stated "ontology research is database research for the $21^{\text {st }}$ century where data need to be shared and not always fit into a simple table".

\subsection{Types of ontologies}

Over the years, researchers of this body of knowledge, tried to clarify, classify and typify the concept of ontology, in terms of its definition, components, and application areas. It seems to have some consensus that the types of ontologies, by subject or content matter are:

- Domain or content ontology - represents the knowledge valid for a given type of domain (e.g. enterprise, medical, electronic, mechanic).

- Meta-data ontology - provide a vocabulary for describing informational content (e.g. Dublin core describes on-line information sources).

- General or common-sense ontology - provides basic notions and concepts about describing general knowledge about the world and so they are valid across several domains (e.g. time, space, state, event).

- Representational/frame ontology - ontologies that provide representational entities without stating what particular domain it represents. Do not commit to any particular domain.

- Task/method/problem solving ontology - provide terms specific for particular tasks and problem-solving methods. It defines primitives by which the problem solving context can be described and domain knowledge can be put into the problem solving context.

\section{Knowledge repository framework}

Knowledge repositories (KR) are a crucial component for the sharing, reuse and knowledge transfer, and thus for improving the performance of any organization. The intermediary of crowdsourcing innovation requires a KR to represent and integrate all the knowledge created by its processes.

Knowledge repositories are a subject that has been studied for a long time by researchers. However, KR's implementation projects still fail in assuring its effective use by employees of the organization, sharing and reuse of knowledge. The major difficulties identified in literature [13] are:

- the weak incentives for sharing knowledge given by the organizations;

- the quantity of information returned by the repository, motivated by bad choices in the design of the repository;

- the cost associated with projects implementing repositories; 
- $\quad$ and to specify the requirements for reuse and sharing of knowledge in each situation and its implications for the design of the repository and related interventions.

So, it is necessary a KR framework based on the real needs and motivations of the potential users of the repository, which implements effective strategies for retrieval and dissemination of knowledge and that also analyze the value and usefulness of content provided by the KR.

The ontologies are presented as a conceptual model for the systematization and formalization of consensual knowledge in a field of knowledge. This conceptualization is rendered concrete with the definition of terms and concepts from the domain of knowledge in analysis, their relationships, organization and hierarchy, and allows the sharing and reuse by different people and systems of such knowledge [14-20]).

Various ontologies have emerged, particularly in the areas of business and enterprise (cf [14], [15], [21], [16], [17]). [22], [23], and more recently [24], proposed ontologies for the process of innovation management, but they represent only the component relating to the process of generating ideas. At the best of our knowledge, there is no ontology defined that represents the entire process of creating an intermediate value of crowdsourcing innovation.

Thus, an ontology of crowdsourcing innovation for intermediaries will be an useful instrument to understand this phenomenon and thus will also be a facilitator for the emergence of such intermediaries. Regarding the types of ontologies described in the previous section we are going to develop a domain ontology to represent the crowdsourcing innovation body of knowledge.

Based on the crowdsourcing innovation intermediary framework presented by Ramos et al. [7] we developed a generic sketch of the conceptual framework for the KR (Fig. 1). 


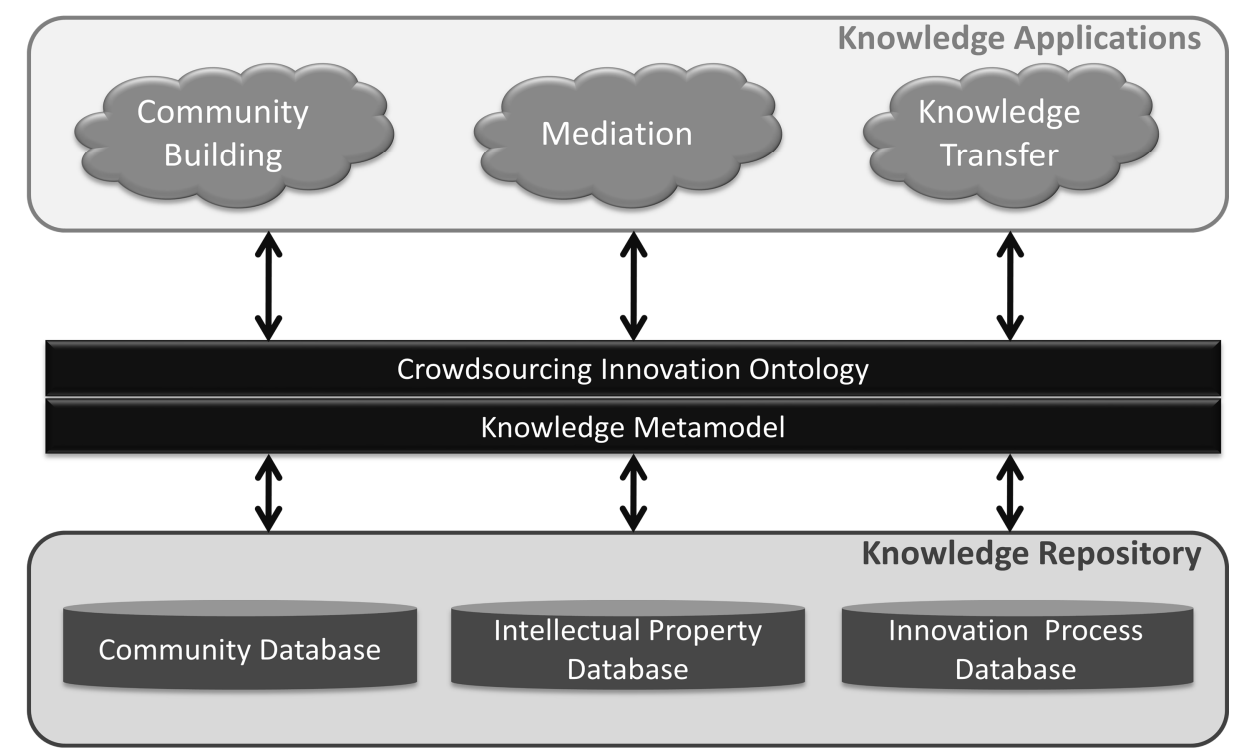

Fig. 1 - Generic conceptual KR framework

The main characteristics of this framework are (1) the construction of collective memory of the community, and the storage of explicit knowledge captured and exchanged by the various online learning activities and social matters; (2) to capture and to disseminate explicit knowledge created and exchanged in the activities of intermediation, such as contract negotiation, project management, marketing, and intellectual property management; (3) capturing and making available the knowledge created in the process of technology transfer, supporting the development of acquired technology in commercial or organizational high-value innovations; and (4) guarantee interoperability between different systems of crowdsourcing innovation.

A crowdsourcing innovation broker can benefit from a structured and integrated KR that allows managing information and knowledge created by the three value creation processes: knowledge community building, intermediation, and technology incubation.

The KR will be both the enabler of the community's collective memory and the repository of the explicit knowledge captured and exchanged in the various learning and social activities online. The knowledge services of this module are centered in providing access to the crowd by tracking and profiling users, managing and moderating virtual communities, and creating proper incentives, and evaluating ideas and solutions posted. The KR will also capture explicit knowledge created and exchanged in the activities of intermediation, such as contract negotiation, project management, challenges delivery and IP commercialization. The knowledge transfer module will facilitate the diagnosis of the seeker and the commercialization of the solutions. It will gather, store and manage knowledge about activities that assist in the transfer of IP acquired in a business or organizational innovation, including specific consulting, tracking of funding and partnerships opportunities, making information on 
market trends available, and supporting the management of innovation projects for the corporate clients.

\section{Conclusion and Future work}

The intermediation of crowdsourcing innovation is a recent research subject that promises to be a way of helping companies to access external innovative ideas and solutions to take advantage of structured knowledge repositories and to support their networking efforts along the value chain.

Knowledge and information in a company augment expressively every day, becoming increasingly difficult to integrate them in an effective way for the decisionmaking process. Besides being available, knowledge must be disseminated by people in the organization before they need it, so that the organization can anticipate events rather than reacting to them.

Knowledge repositories are a crucial component for the sharing, reuse and transfer of knowledge, and thus for improving the performance of any organization. Although being a subject that has been studied for a long time by researchers, KR's implementation projects still fail in assuring its effective use, sharing and reuse of knowledge by employees of the organization.

This research project intends to contribute to leverage KR usage and helpfulness to the decision-making process by proposing a new conceptual architecture of KR, specially guided to crowdsourcing innovation intermediaries.

The development of the KR architecture will be sustained by the development of a crowdsourcing innovation ontology. Ontologies have been developed with the promise of providing knowledge sharing and reuse between people and systems, by building a conceptual framework of a given knowledge domain to be represented. This framework is a consensus conceptualization of a given domain, which permit the accomplishment of interoperability between systems and persons.

Thus, the development of the ontology will be an instrument to help to understand the phenomenon of crowdsourcing innovation, and will also be a facilitator for the emergence of such intermediaries.

\section{Acknowledgments}

To the Foundation for Science and Technology and the Polytechnic Institute of Oporto for financial support (SFRH/BD/49263/2008) of this research project.

\section{References}

1. D. Tapscott and A. D. Williams, Wikinomics: How Mass Collaboration Changes Everything. Portfolio, 2008.

2. H. H. Chesbrough, "The era of open innovation," MIT SLOAN MANAGEMENT REVIEW, vol. 14 , no. 3, pp. 35-41, Spring.

3. H. W. Chesbrough, Open Innovation: Researching a New Paradigm, Henry Chesbrought,Wim Vanhaverbeke and Joel West, Eds. Oxford University Press, 2006. 
4. M. W. Wallin and G. Von Krogh, "Organizing for Open Innovation:: Focus on the Integration of Knowledge," Organizational Dynamics, vol. 39, no. 2, pp. 145-154, 2010.

5. J. Howe, Crowdsourcing: How the Power of the Crowd is Driving the Future of Business. Crown Business, 2008.

6. C. Adams and I. Ramos, "Crowdsourcing: A Social Networking Approach to Outsourcing," Cutter IT Journal, vol. 22, no. 10, 2009.

7. I. Ramos, M. Cardoso, J. Carvalho, and J. Graça, "An Action Research on Open Knowledge and Technology Transfer," in Information Systems - Creativity and Innovation in Small and Medium-Sized Enterprises, vol. 301, Springer Boston, 2009, pp. 211-223.

8. M. Hepp, "Ontologies: State of Art, Business Potential, and Grand Challenges," in Ontology Management: Semantic Web, Semantic Web Services, and Business Applications, Martin Hepp. Pieter De Leenheer, Aldo de Moor, York Sure. (Eds)., Springer, 2007, pp. 3-22.

9. T. R. Gruber, "A translation approach to portable ontology specifications," Knowledge Aquisition, no. 5, pp. 199-220, 1993.

10. D. Gasevic, D. Djuric, and V. Devedzic, Model Driven Architecture and Ontology Development. Springer-Verlag Berling Heidelberg, 2006.

11. D. Fensel, Ontologies: A Silves Bullet for Knowledge Management and Electronic Commerce, 2nd ed. Springer-Verlag Berling Heidelberg, 2004.

12. J. Howe, "The Rise of Crowdsourcing," Wired Magazine, vol. 14.06, pp. 1-4, Jun-2006.

13. L. M. Markus, "Toward a Theory of Knowledge Reuse: Types of Knowledge Reuse Situations and Factors in Reuse Success," Journal of Management Information Systems, vol. 18 , no. 1, pp. 57-93, 2001.

14. Ó. Corcho, M. Fernández-López, A. Gómez-Pérez, and Ó. Vicente, "WebODE: An Integrated Workbench for Ontology Representation, Reasoning, and Exchange," in Knowledge Engineering and Knowledge Management: Ontologies and the Semantic Web, 2002, pp. 295-310.

15. D. Fensel, F. Van Harmelen, M. Klein, and H. Akkermans, "On-To-Knowledge: Ontology-based Tools for Knowledge Management," in PROCEEDINGS OF THE EBUSINESS AND EWORK 2000 (EMMSEC 2000) CONFERENCE, 2000.

16. A. Smirnov, M. Pashkin, N. Chilov, and T. Levashova, "Ontology-based knowledge repository support for healthgrids," Studies of technology and informatics, vol. 112, pp. 47-56, 2005.

17. L. Tang et al., "WISE: A Prototype for Ontology Driven Development of Web Information Systems," in Frontiers of WWW Research and Development - APWeb 2006, vol. 3841/2006, Springer Berlin / Heidelberg, 2006, pp. 1163-1167.

18. M. Uschold, M. King, S. Moralee, and Y. Zorgios, "The Enterprise Ontology," The Knowledge Engineering Review, vol. 13, no. 1, pp. 31-89, 1998.

19. M. Leppänen, "A Context-Based Enterprise Ontology," in Business Information Systems, 2007, vol. 4439/2007, pp. 273-286.

20. M. F. Lopez, A. Gomez-Perez, J. P. Sierra, and A. P. Sierra, "Building a chemical ontology using Methontology and the Ontology Design Environment," Intelligent Systems and their Applications, IEEE, vol. 14, no. 1, pp. 37-46, 1999.

21. M. Hepp, "Possible Ontologies: How Reality Constrains the Development of Relevant Ontologies," IEEE Internet Computing, vol. 11, no. 1, pp. 90-96, 2007.

22. K. Ning, D. O'Sullivan, Q. Zhu, and S. Decker, "Semantic innovation management across the extended enterprise," International Journal of Industrial and Systems Engineering, vol. 1, no. 1-2, pp. 109-128, 2006.

23. A. C. Bullinger, Innovation and Ontologies. Gabler Verlag, 2008.

24. C. Riedl et al., "An Idea Ontology for Innovation Management," International Journal on Semantic Web and Information Systems, vol. 5, no. 4, pp. 1-18, 2009. 